OPEN ACCESS

Edited by:

Mingyan Zhu,

Affiliated Hospital of Nantong

University, China

Reviewed by:

Yikang $L u$,

Changzhi Medical College, China

Xu Lifen,

Soochow University, China

${ }^{*}$ Correspondence:

Zan Fu

fuzan1971@njmu.edu.cn

${ }^{\dagger}$ These authors have contributed equally to this work

Specialty section:

This article was submitted to Molecular and Cellular Oncology,

a section of the journal

Frontiers in Cell and Developmental

Biology

Received: 20 January 2021

Accepted: 24 March 2021

Published: 15 April 2021

Citation:

Shen H, Huang C, Wu J, Li J, Hu T, Wang Z, Zhang $H$, Shao $Y$ and

Fu Z (2021) SCRIB Promotes Proliferation and Metastasis by Targeting Hippo/YAP Signalling

in Colorectal Cancer.

Front. Cell Dev. Biol. 9:656359. doi: 10.3389/fcell.2021.656359

\section{SCRIB Promotes Proliferation and Metastasis by Targeting Hippo/YAP Signalling in Colorectal Cancer}

\author{
Hengyang Shen ${ }^{1 t}$, Changzhi Huang ${ }^{1 t}$, Jingyu Wu ${ }^{1+}$, Jie Lit', Tao Hu${ }^{2}$, Zhenling Wang ${ }^{1}$, \\ Hongqiang Zhang ${ }^{1}, \mathrm{Yu}$ Shao ${ }^{1}$ and Zan Fu' ${ }^{1 *}$ \\ ${ }^{1}$ Department of General Surgery, The First Affiliated Hospital of Nanjing Medical University, Jiangsu Province Hospital, \\ Nanjing, China, ${ }^{2}$ Department of General Surgery, The Affiliated Jiangning Hospital of Nanjing Medical University, Nanjing, \\ China
}

The complex in which scribble planar cell polarity protein $(S C R / B)$ is located is one of the three main polar protein complexes that play an important role in maintaining epithelial polarity and affecting tumour growth. However, the role of SCRIB in colorectal cancer (CRC) remains largely unknown. This study used date from The Cancer Genome Atlas (TCGA) and clinical samples to determine the expression of SCRIB in CRC and explored its mechanism through bioinformatics analysis and in vivo and in vitro experiments. In this study, SCRIB was found to be highly expressed in CRC patients, and it was often associated with malignant characteristics, such as proliferation, apoptosis, and epithelial-mesenchymal transition (EMT). Furthermore, we found that SCRIB may interact with the Hippo signalling pathway and affect the phosphorylation of YAP and its distribution inside and outside of the nucleus. We concluded that increased expression of SCRIB is likely to inhibit the Hippo signalling pathway by promoting YAP phosphorylation. This role of SCRIB in the progression of CRC provides an important information for the treatment of CRC.

Keywords: colorectal cancer, SCRIB, Hippo signaling pathway, tumour invasion and poor prognosis, cell cycle, cell polarity

\section{INTRODUCTION}

Colorectal cancer (CRC) is the third most common malignant tumour with the third highest incidence and the second highest mortality rate worldwide, and the age of patients at CRC onset is decreasing (Siegel et al., 2020a,b). The increase in the incidence of CRC among Chinese population in the past decade is closely related to the changes in people's living habits due to rapid economic development; these changes include unhealthy eating, drinking, smoking, lack of exercise and obesity (Zhu et al., 2017). Although the use of surgery and comprehensive treatment has benefited many CRC patients, nearly half of the patients who undergo radical surgery may relapse, and patients who die from CRC liver metastases account for more than 50\% of all CRC-related deaths

Abbreviations: SCRIB, scribble planar cell polarity protein; CRC, colorectal cancer; TCGA, The Cancer Genome Atlas; YAP, Yes1 associated transcriptional regulator; PCR, polymerase chain reaction; qRT-PCR, quantitative real-time polymerase chain reaction; RT-PCR, reverse transcription polymerase chain reaction; EDU, 5-Ethynyl-2'-deoxyuridine; CCK-8, Cell Counting Kit 8; IHC, immunohistochemistry; IF, Immunofluorescence; PBS, phosphate buffer saline; TNM, tumour-node-metastasis; EMT, epithelial-mesenchymal transition; LPA, lysophosphatidic acid. 
(Enomoto and Igaki, 2011; Beckers et al., 2018). The occurrence of CRC is an extremely complicated process that may involve biological behaviours such as cell proliferation, metabolism, apoptosis, invasion and metastasis (Hanahan and Weinberg, 2000, 2011). However, the specific mechanisms underlying the occurrence and distant metastasis of CRC have yet to be determined.

The development of solid tumours is actually an process driven by internal and external factors that causes normal cells to proliferate in an unregulated manner and to acquire migratory and invasive capabilities (Hanahan and Weinberg, 2011). In particular, disordered cell polarity is one of the important intracellular factors that is important for the malignant progression of CRC and other epithelial cell tumours (Rothenberg et al., 2010; St Johnston and Ahringer, 2010; Muthuswamy and Xue, 2012; Chatterjee and McCaffrey, 2014). Scribble complexes, which are one of the three types of polar complexes, have a profound impact on the apical-basal polarity of epithelial cells (Assémat et al., 2008; Lee and Vasioukhin, 2008). In several studies, the SCRIB gene, which encodes the Scribble protein, is even considered to be a regulator of tumour development and metastasis due to its role in tumour-related mechanisms (Zen et al., 2009; Royer and Lu, 2011). However, considering that the function of SCRIB in different, even opposite, in different tumours, it cannot be generally considered to be a cancer-promoting or cancer-suppressing gene (Zhan et al., 2008; Feigin et al., 2014). Our previous study found that there is a polymorphic site in SCRIB, namely rs13251492 $\left(P=7.76 \times 10^{-5}\right)$, that is significantly associated with CRC, and we proved that this genetic variation can affect the expression of SCRIB (Shen et al., 2020). However, the impact of SCRIB on the progression of CRC and its underlying mechanism are not yet known.

Our study determined that SCRIB is highly expressed in CRC and indicates a poor prognosis. We performed gain-of-function and loss-of function experiments and found that SCRIB promotes the proliferation, invasion and migration of CRC, and inhibits its apoptosis in vitro and in vivo. The bioinformatics prediction and the corresponding verification experiments together yielded the result that SCRIB progression of CRC by influencing the Hippo signalling pathway. Therefore, our study elucidates the mechanism by which SCRIB participates in CRC and may provide a new target for the treatment of CRC and a new perspective for clinical decision-making.

\section{MATERIALS AND METHODS}

\section{Clinical Samples and Ethical Approval}

Human CRC and adjacent normal mucosa samples were obtained from CRC patients who underwent surgery at the First Affiliated Hospital of Nanjing Medical University between 2014 and 2018. Within 5 min of surgical resection, the samples were immediately embedded in the paraffin or transferred to the $-80^{\circ} \mathrm{C}$ freezer. All clinicopathological diagnoses were confirmed by at least two pathologists according to the guidelines of the American Joint Committee on Cancer. All experiments were approved by the Ethics Committee of the First Affiliated Hospital of Nanjing Medical University, and informed consent was obtained from the all the patients before enrolment in this study.

\section{Cell Culture and Transfections}

Normal epithelial colon cells (NCM460 cells) and human CRC cells, including Lovo, HCT116, SW620, DLD-1 and HT-29 cells, were obtained from American Type Culture Collection. RPMI1640 medium and McCoy'5A medium (HyClone, Logan, UT, United States) were mixed with $10 \%$ fetal bovine serum to culture the DLD-1 and HCT116 cells, respectively. The cells were cultured in a humid incubator, which always maintained a temperature of $37^{\circ} \mathrm{C}$ and an atmosphere of $5 \% \mathrm{CO}_{2}$. The siRNAs targeting SCRIB and the corresponding negative controls (si-NC) were synthesized by RiboBio (Guangzhou, China). The siRNA target sequence (GAAGCAGCTATCCATCCTA) with the highest efficiency for knocking down the expression of SCRIB was cloned into the lentivirus vector pGLV3 to by GeneChem (Shanghai, China). GeneChem Company designed and constructed the SCRIB overexpression plasmid for our study. Lipofectamine 3000 (Invitrogen) was used for the transfection of cells.

\section{RNA Extraction and Polymerase Chain Reaction (PCR)}

Total RNA was extracted from CRC tissue and CRC cell lines with TRIzol reagent according to the manufacturer's instructions (Qiagen, Hilden, Germany). The HiScript ${ }^{\circledR}$ III RT SuperMix (Vazyme, Nanjing, China) was used to reverse transcribe the extracted total RNA into cDNA by reverse transcription PCR (RT-PCR). The genes were amplified and their expression levels were detected by quantitative real-time PCR (qRT-PCR) using ChamQ SYBR qPCR Master Mix (Vazyme, Nanjing, China). The results were normalized to the expression levels of GAPDH. The primers for the target mRNAs and internal control were as follows: SCRIB, forward primer, 5'-CCTCTGTCAAGGGAGTGTCG- $3^{\prime}$ and reverse primer, $5^{\prime}$-CCCGAGAGATGAATATGCCCTC-3'; YAP, forward primer, 5'-TAGCCCTGCGTAGCCAGTTA-3', reverse primer, 5'TCATGCTTAGTCCACTGTCTGT-3'; GAPDH, forward primer, 5'-GGAGCGAGATCCCTCCAAAAT-3', reverse primer, 5'-GGCTGTTGTCATACTTCTCATGG-3'; ZO-1, forward primer, CGGTCCTCTGAGCCTGTAAG, reverse primer, GGAT CTACATGCGACGACAA; E-cadherin, forward primer, 5'-AC CAGAATAAAGACCAAGTGACCA-3', reverse primer, 5' AGCAAGAGCAGCAGAATCAGAAT-3'; N-cadherin, forward primer, 5'-GGACAGTTCCTGAGGGAT CA-3', reverse primer, 5'-GGATTGCCTTCCATGTCTG T-3'; Vimentin, forward primer, 5'-GATGCGTGAGATGGAAGAGA-3', reverse primer, 5'-GGCCATGTTAACATTGAGCA-3'.

\section{Western Blot Analysis}

Cells and tissue fragments were sonicated and then RIPA lysis buffer (Beyotime, Shanghai, China) was used for protein extraction. The concentrations of the purified proteins were 
detected by bicinchoninic acid (BCA) protein assay (Beyotime, Shanghai, China). The protein samples were separated by $10 \%$ gels using SDS-PAGE and transferred to PVDF membranes (Millipore, Billerica, MA, United States). The antibodies against $S C R I B$, E-cadherin, ZO-1, N-cadherin, Vimentin, Histone H3, $\mathrm{Ki67}, \mathrm{Cdk} 4, \mathrm{GAPDH}$ and $\beta$-actin were purchased from Abcam (Cambridge, MA, United States). The antibodies against YAP and phospho-YAP (Ser127) were purchased from Cell Signaling Technology (Danvers, MA, United States).

\section{Scratch Wound Healing Assay and Transwell Assay}

The cells were seeded in a 6-well plate at a density of $4 \times 10^{5}$ cells/well and cultured until the cells covered almost the entire well. A $200-\mu \mathrm{L}$ pipette tip was used to scrape a linear wound. The culture medium in the dish was replaced with serum-free medium at 0 and 24 hours after injury, and the migrating cells at the edge of the wound were monitored by an inverted microscope. According to the manufacturer's instructions, Millicell cell culture inserts (24-well inserts with $8-\mu \mathrm{m}$ pores) were used for Transwell and invasion analyses. To perform the migration assays, the bottom of the insert was seeded with $4 \times 10^{4}$ cells (per well) in $200 \mu \mathrm{L}$ of serum-free medium, and then, $500 \mu \mathrm{l}$ of medium containing 10\% FBS was added to the lower chamber. For the invasion assay, Matrigel (BD Biosciences, Franklin Lakes, NJ, United States) was added to the insert, and then, $8 \times 10^{4}$ cells (per well) were plated in serum-free medium. Add Medium containing 10\% FBS was added to the lower chamber. After 24 hours of incubation, the cells on the bottom of the membrane were fixed and stained with $0.5 \%$ crystal violet solution. We used light microscopy to observe the migrating and invading cells and randomly selected 3 fields for counting.

\section{Cell Proliferation Assay}

For the colony formation assay, cells were plated in 6-well plates. We counted the number of colonies 14 days later and photographed representative wells. In addition, a cell proliferation assay was also performed with the 5-ethynyl-2'deoxyuridine assay (EDU) Kit (Beyotime, Shanghai, China) and Cell Counting Kit 8 (CCK-8) assay (Beyotime, Shanghai, China) according to the manufacturer's protocols. The detailed methods were described in our previous research (Hu et al., 2020).

\section{Immunohistochemistry}

Immunohistochemistry (IHC) was conducted as previously described (Hu et al., 2020). Tissue sections with a thickness of $4 \mu \mathrm{m}$ were first deparaffinized with xylene and then rehydrated with gradient alcohol. The samples were incubated with $3 \% \mathrm{H}_{2} \mathrm{O}_{2}$ for $10 \mathrm{~min}$ at room temperature to block the endogenous peroxidase activity. Finally, the primary antibodies were combined with the proteins in the sample by using the streptavidin peroxidase-conjugated method. Photographs of the sections were obtained using a digital microscope camera. The staining score was used to indicate the expression level of genes, and the specific scoring rules can refer to previous studies.

\section{Immunofluorescence (IF)}

The transfected cells were fixed with $4 \%$ paraformaldehyde for $20 \mathrm{~min}$. The cells were incubated in $0.1 \%$ Triton X-100 for 4 hours at room temperature to achieve permeabilization. The cells were blocked in the blocking solution for $60 \mathrm{~min}$ and gently shaken on a shaker. The blocking solution was removed by washing with phosphate-buffered saline (PBS), and the samples were incubated with diluted primary antibodies against YAP (1:250; Abcam) for $60 \mathrm{~min}$. The primary antibodies were removed, and the cells were washed 3-5 times with PBS for 3-5 min each time. After removing the washing solution, $1 \mathrm{ml}$ of Alexa Fluor488-conjugated secondary antibodies (Beyotime, Shanghai, China) was added and incubated for $60 \mathrm{~min}$ in the dark. Fluorescence confocal images was captured by confocal fluorescence microscopy (Zeiss Germany, Germany).

\section{Animal Experiments}

We chose to use 6- to 8-week-old $\mathrm{BALB} / \mathrm{c}$ nude male mice for the subcutaneous tumour formation experiments. For the tumorigenicity studies, The HCT116 cells stably transfected with lentivirus and DLD-1 cells transfected with the overexpression plasmid were used. A total of $5 \times 10^{6}$ of the indicated cells, including control cells, were subcutaneously injected into the left and right axilla of each nude mouse. The volume and weight of the tumours were measured every 5 days. After 4-6 weeks, xenograft tumours were dissected from sacrificed nude mice and used for IHC. All the animal experiments were approved by the Committee on the Ethics of Animal Experiments of Nanjing Medical University.

\section{Cell Cycle and Apoptosis Analysis}

For cell cycle analysis, we use the appropriate methods to process the cells and set up a negative control group. A total of 1-10 $\times 10^{5}$ cells were washed with PBS and centrifuged at 2,000 rpm per minute. Then, $500 \mu \mathrm{l}$ of cold $70 \%$ ethanol was added to the precipitate for fixation, and the samples were incubated overnight at $4^{\circ} \mathrm{C}$. Before staining, we washed away the fixative with PBS, added $100 \mu \mathrm{l}$ RNase A (KeyGene, Jiangsu, China) and then incubated the samples at $37^{\circ} \mathrm{C}$ for $30 \mathrm{~min}$. Then, $400 \mu \mathrm{l} \mathrm{PI}$ was added to the tubes, and the samples were incubated at $4^{\circ} \mathrm{C}$ in the dark for $30 \mathrm{~min}$. Finally, flow cytometry analysis was performed according to the protocol. The apoptosis assay was performed using an Annexin V-FITC/PI (MultiScience, Zhejiang, China). The cells were digested and centrifuged at 1,500 rpm for $5 \mathrm{~min}$. The cells were centrifuged and washed with prechilled PBS. Approximately $1-10 \times 10^{5}$ cells were collected, including the cells in the culture supernatant. Five hundred microlitres of the working solution diluted in double-distilled water was to resuspend the cells. A total of $5 \mu \mathrm{l}$ Annexin V-FITC and $10 \mu \mathrm{l}$ PI were added to each tube. After gentle vertexing, the cells were incubated for $5 \mathrm{~min}$ at room temperature in the dark. Finally, flow cytometry analysis was performed according to the protocol.

\section{TUNEL Staining}

According to the manufacturer's protocol, a one-step TUNEL (TdT-mediated dUTP gap end labelling) apoptosis detection kit 
(Beyotime, C1090) was used to examine the apoptosis of HCT116 and DLD-1 cells after the knockdown and overexpression of $S C R I B$, respectively. The cells were photographed under an Olympus FSX100 microscope (Tokyo, Japan).

\section{Statistical Analysis}

All the experiments were carried out at least three independent times. All the statistical analyses were performed using Social Sciences 22.0 software (SPSS, CA, United States), and the statistical results were visualized using GraphPad Prism 8.0 (GraphPad Software, CA, United States). The $t$-test was used to compare the differences between the two groups of data that conformed to the Gaussian distribution, while the Wilcoxon test and the Mann-Whitney test were used for the paired and unpaired data with a the non-Gaussian distribution. One-way ANOVA was used to compare the differences between multiple groups of data, and the $\chi^{2}$ test was used to compare categorical variables. All the data are presented as the mean \pm SD (standard deviation) of triplicates. $P$ values $<0.05$, as determined using two-sided tests, were considered statistically significant.

\section{RESULTS}

\section{Upregulation of SCRIB in CRC Predicts a Poor Prognosis}

In our previous research, a large amount of data from TCGA and the Gene Expression Omnibus database was analysed to illustrate the relationship between SCRIB and CRC (Shen et al., 2020). Considering the availability of updated data, we repeated some of these significant analyses. Our new analysis showed that the mRNA level of SCRIB in tumour tissues was significantly increased compared to that in normal colorectal tissues $\left(P=2.51 \times 10^{-20}\right)$ (Figure 1A). In addition, the survival analysis results showed that CRC patients with high SCRIB expression were more likely to relapse (Figure 1B). In the stratified analysis of tumour tissue, we found that the different pathological types of CRC, adenocarcinoma and mucinous adenocarcinoma, had obvious differences $\left(P=8.32 \times 10^{-8}\right)$. The expression level of $S C R I B$ was higher in advanced patients with tumour-node-metastasis (TNM) staging, and high SCRIB expression was also observed in the CRC cancer tissue of patients with metastasis and lymphatic invasion (Figure 1C). Together, these results indicate that the expression of SCRIB may have potential value for predicting the progression of CRC. Therefore, we further detected the expression level of SCRIB in CRC tissues and analysed the relationship between its expression level and the epidemiological and pathological characteristics of CRC patients (Table 1). The expression of SCRIB did not change due to the age, sex, and living habits of CRC patients, but there were indeed differences in patients with different TNM stages $(P=0.018)$, this observation is consistent with the TCGA analysis results. The qRT-PCR and western blot results showed that SCRIB substantially upregulated in tumours tissues compared with the adjacent nontumorous tissues at both the mRNA and protein levels (Figures 1D,E). The high expression of SCRIB in CRC tissues was also shown by IHC staining of CRC tissues
(Figure 1F). The patients were divided into two groups based on high SCRIB expression and low SCRIB expression to study the relationship between the expression level of $S C R I B$ and the clinical characteristics of CRC patients (Table 1). In addition, the expression of SCRIB in various CRC cells was higher than that in normal colorectal cells (Figure 1G).

\section{SCRIB Upregulation Enhanced the Metastatic Capacity of CRC}

Based on the differential expression of SCRIB in different CRC cells, we performed loss-and gain-of-function assays with HCT116 cells, which have high SCRIB expression, and DLD1 cells, which have low SCRIB expression (Figure 1G). First, the knockdown lentivirus and overexpression plasmids were transfected into HCT116 and DLD-1 cells, respectively. The knockdown and overexpression efficiencies were examined by qRT-PCR and western blot respectively (Figures 2A,B). Considering the distribution of SCRIB expression in patients with different TNM stages, distant metastasis and lymphatic invasion (Figure 1C), we have a strong interest in studying the impact of SCRIB on the invasion and metastasis of CRC cell lines. The wound healing assays demonstrated that SCRIB downregulated inhibited the migration of HCT116 cells and SCRIB overexpression significantly increased the migration of DLD-1 cells compared to that of control cells (Figure 2C). Transwell assays showed that downregulated SCRIB reduced the migration and invasion of HCT116 cells relative to those of control cells. Correspondingly, DLD-1 cells overexpressing SCRIB showed enhanced cell migration and invasion capabilities (Figure 2D). We used qRT-PCR and western blotting to detect the expression levels of several epithelial-mesenchymal transitions (EMT) markers to further verify whether SCRIB promotes CRC transfer by regulating EMT. ZO-1 is a tight junction protein, E-cadherin is an epithelial marker, and $\mathrm{N}$-cadherin and vimentin are mesenchymal markers. The results of Figure 2E show that the knockdown of SCRIB in HCT116 cells increased the expression of ZO-1 and E-cadherin and decreased the expression of the $\mathrm{N}$-cadherin and vimentin at the mRNA and protein levels (Figure 2E). In contrast, DLD1 cells overexpressing $S C R I B$ showed a significant decrease in the expression of E-cadherin and ZO1 and an increased in the expression of vimentin and $\mathrm{N}$-cadherin (Figure 2F).

\section{The Expression of SCRIB Affects the Proliferation and Cell Cycle of CRC Cells}

We further studied the role of SCRIB in CRC cell proliferation and cell cycle progression. First, we used a CCK- 8 assay to study the effect of SCRIB on the proliferation of CRC cells by observing the absorbance of the cells for 5 consecutive days. The results of the CCK-8 experiment showed that the proliferation ability of HCT116 cells with downregulated SCRIB was reduced, while the upregulation of $S C R I B$ enhanced the proliferation ability of DLD-1 cells (Figure 3A). Colony formation assays showed that HCT116 cells with SCRIB knocked down formed fewer colonies than control cells, while DLD-1 cells overexpressing SCRIB formed more colonies than control cells (Figure 3B). The results 
A

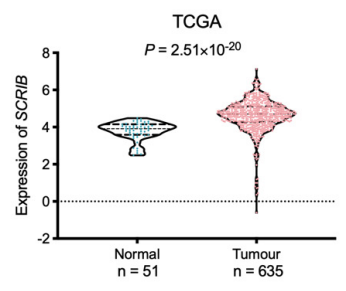

B

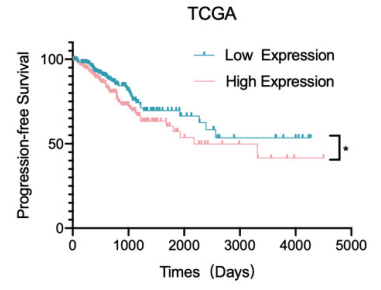

D

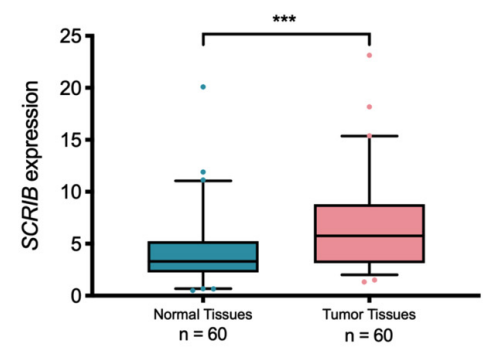

E
C
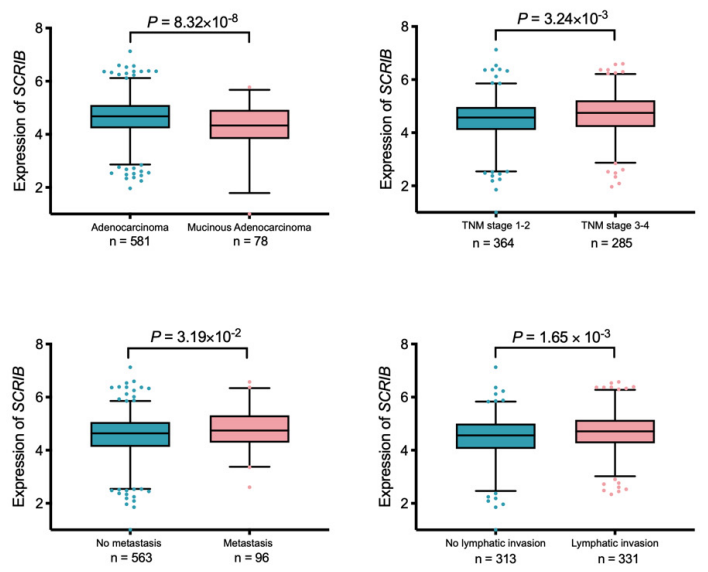

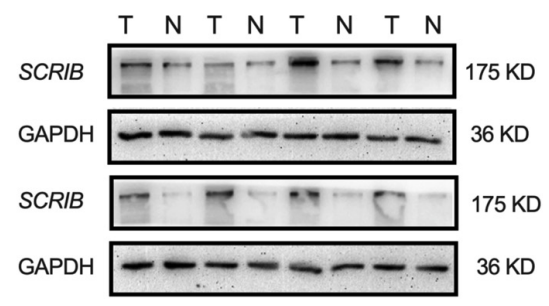

$\mathbf{F}$

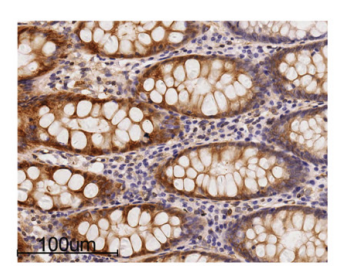

Normal Tissues

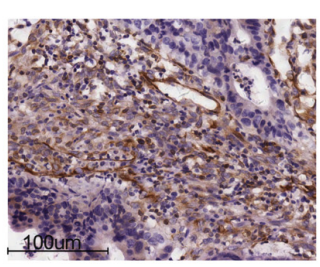

CRC Tissues

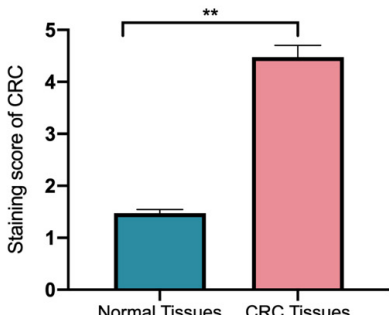

G
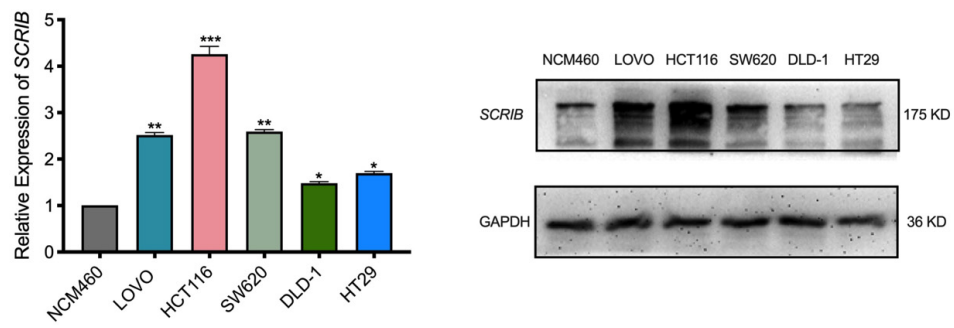

FIGURE 1 | Upregulation of SCRIB predicts poor prognosis for patients with colorectal cancer. (A) The expression level of $S C R I B$ in normal tissues $(n=51)$ and CRC tissues $(n=635)$ from The Cancer Genome Atlas (TCGA) database. (B) CRC patients $(n=499)$ with progression-free survival data in the TCGA database were included in the survival analysis. The population was divided into two groups according to the median expression level of SCRIB. (C) The stratified analysis of CRC patients was based on pathological type, stage, metastasis, and lymphatic invasion. The data were derived from TCGA. Due to the lack of patient data, the total number of stratified analyses is not consistent. (D) SCRIB expression in 60 paired CRC tissues and adjacent normal tissues. (E) The expression of SCRIB in eight pairs of CRC tissues (T) and adjacent normal tissues (N) was detected by western blot. (F) The expression of SCRIB in CRC tissue was assessed by immunohistochemical staining. Representative micrographs show high-level and low-level expression of SCRIB in tumour tissues and adjacent nontumorous tissues. Scale bar, $100 \mu \mathrm{m}$. (G) qRT-PCR showed the expression level of SCRIB in NCM460, LOVO, HCT116, SW620, DLD-1, and HT29 cells; western blot showed the protein level of SCRIB in these cell lines. ${ }^{*} p<0.05,{ }^{* *} p<0.01$, and ${ }^{* * *} p<0.001$. 
TABLE 1 | Relevance analysis of SCRIB expression in CRC patients.

\begin{tabular}{|c|c|c|c|c|c|}
\hline \multirow[t]{2}{*}{ Variables } & \multirow[t]{2}{*}{ All patients } & \multicolumn{2}{|c|}{ SCRIB Level } & \multirow[t]{2}{*}{$x^{2}$} & \multirow[t]{2}{*}{$P^{a}$} \\
\hline & & Low & HIGH & & \\
\hline All Cases & 60 & 30 & 30 & & \\
\hline Age (years) & & & & 0.317 & 0.573 \\
\hline$<60$ & 18 & 10 & 8 & & \\
\hline$\geq 60$ & 42 & 20 & 22 & & \\
\hline Gender & & & & 0.278 & 0.598 \\
\hline Male & 36 & 17 & 19 & & \\
\hline Female & 24 & 13 & 11 & & \\
\hline Smoking status & & & & 0.278 & 0.598 \\
\hline Never & 36 & 19 & 17 & & \\
\hline Ever & 24 & 11 & 13 & & \\
\hline Drinking status & & & & 1.27 & 0.26 \\
\hline Never & 42 & 23 & 19 & & \\
\hline Ever & 18 & 7 & 11 & & \\
\hline Tumor location & & & & 0.067 & 0.796 \\
\hline Colon & 29 & 15 & 14 & & \\
\hline Rectum & 31 & 15 & 16 & & \\
\hline Tumor grade & & & & 1.002 & 0.317 \\
\hline Well+Moderate & 49 & 26 & 23 & & \\
\hline Poor & 11 & 4 & 7 & & \\
\hline TNM staging system & & & & 2.443 & $0.018^{*}$ \\
\hline Stage I + II & 35 & 22 & 13 & & \\
\hline Stage III +IV & 25 & 8 & 17 & & \\
\hline
\end{tabular}

${ }^{a}$ Chi-square test was used for comparing groups between low and high SCRIB expression. ${ }^{*} P<0.05$.

of the EDU assays further confirmed that SCRIB can promote cell proliferation (Figure 3C). In light of the function of SCRIB in CRC cell proliferation, we considered whether its expression level would cause differences in the cell cycle distribution and apoptosis of CRC cells. An increased number of cells in the G0 and G1 phases and a decreased number of cells in the $S$ phases were observed in the HCT116 cells with SCRIB-knocked down compared with control cells. In contrast, the overexpression of SCRIB promoted the G1-to-S phase transition in DLD1 cells (Figure 3D). In addition, transfected HCT116 cells exhibited a reduced proportion of apoptotic cells compared to the control cell. The proportion of apoptotic cells in DLD-1 cells overexpressing SCRIB increased significantly (Figure 3E). Based on the results of the above experiments, we further examined the effects of changes in SCRIB expression on cell proliferation and cell cycle progression at the tissue level. In immunohistochemical (IHC) staining, the expression levels of Ki67, CDK4 and Bax were used as markers to detect cell proliferation, cell cycle progression and apoptosis. We observed that SCRIB expression was positively correlated with $\mathrm{Ki} 67, \mathrm{CDK} 4$ and Bax expression at the tissue level (Figure 3F).

\section{SCRIB Contributes to Tumour Proliferation and Progression in vivo}

As shown in the model diagram, HCT116 cells with SCRIB knocked down, DLD-1 cells overexpressing SCRIB, and the corresponding control cells were injected subcutaneously into nude mice (Figure 4A). Tumour growth was markably suppressed by the knockdown of SCRIB as shown by lower final tumour volumes and weights than those of the control tumours, and overexpression of SCRIB promoted tumour growth (Figure 4B). A TUNEL assay was performed, and the results showed that the TUNEL-positive rate in tumour tissues with relatively high SCRIB expression was significantly higher than that in tumour tissues with relatively low SCRIB expression (Figure 4C). Furthermore, we performed immunohistochemical staining of the tumour tissues in nude mice. A decrease in the expression of Ki67 in SCRIB knockdown tumours was observed by IHC staining (Figure 4D).

\section{SCRIB Promotes the Activation of the Hippo/YAP Pathway in CRC Cells}

To further study the mechanism by which SCRIB mediates tumour biological behaviour, we summarized the SCRIB-related molecules, including miRNAs, IncRNAs, transcription factors and interacting proteins, reported in the literature (Figure 5A and Supplementary Table 1). We focused on the proteins related to SCRIB and used the String database to predict the relationships between SCRIB and these proteins. We clearly observed that the protein encoded by $S C R I B$ interacts with most proteins, including those that have been verified by experiments and predicted by bioinformatics (Figure 5B). Then, we analysed the pathway enrichment of these proteins through DAVID, KOBAS, Metascape and GCBI to identify the signalling pathways targeted by SCRIB. Ultimately, the Hippo signalling pathway was revealed to be the only signalling pathway enriched in all four databases (Figure 5C and Supplementary Table 2). CRC data from the TCGA database were used to analyse the correlation between the core genes and downstream target genes of the Hippo signalling pathway and SCRIB (Figure 5D). We first observed that there was no correlation between the expression of SCRIB and YAP, a key gene of the Hippo signalling pathway. However, the target genes TEAD3 and TEAD4, which are activated by YAP in the nucleus, were significantly positively correlated with SCRIB $\left(P=1.50 \times 10^{-8}\right.$ and $P=7.50 \times 10^{-8}$, respectively). Although the expression levels of MST1/2 and SAV1 were not obviously altered as the expression levels of SCRIB increased, LAST1/2 and MOB1A/B were all negatively correlated with $S C R I B$. The activation of the upstream genes of the Hippo signalling pathway, such as MST1/2 and LATS1/2, causes serine 127 of YAP to be phosphorylated, which traps YAP in the cytoplasm to promote transcription (Zhao et al., 2007). Therefore, we assume that the increase in the expression of SCRIB does not directly affect the expression of YAP but rather prevents the LAST1/2 and MOB1A/B complexes from reducing the phosphorylation of YAP, thereby indirectly causing more YAP to enter the nucleus to activate the transcription of the downstream TEAD family of genes. We found by western blot that when SCRIB was knocked down or overexpressed, the protein level of YAP did not change significantly, but the level of phosphorylated YAP 
A

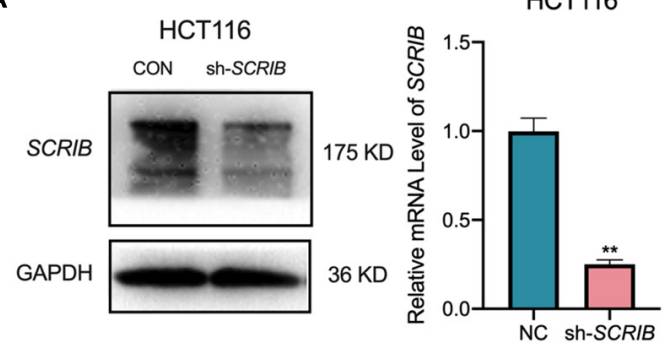

C
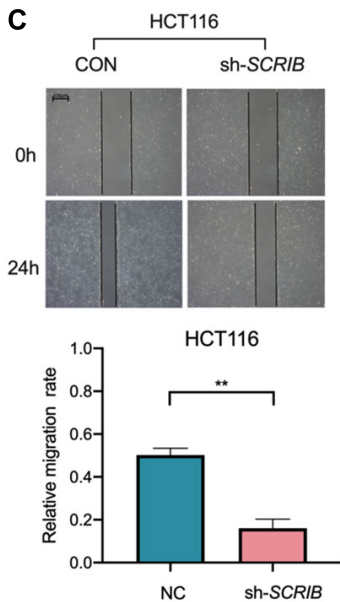

E

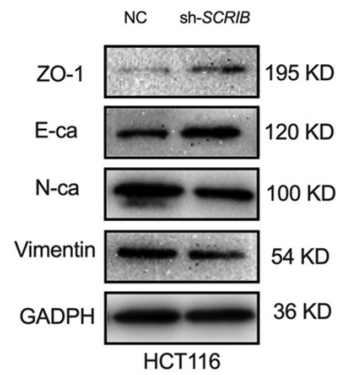

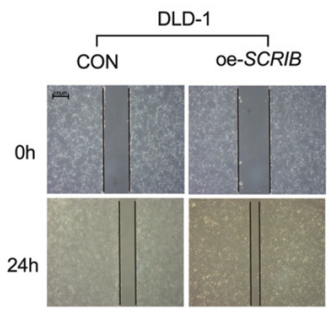

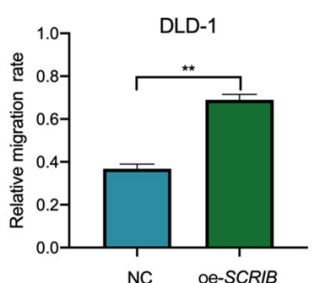

B

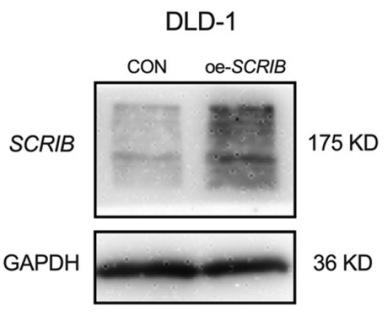

DLD-1

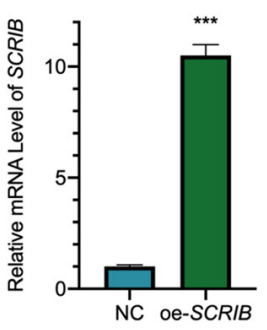

D
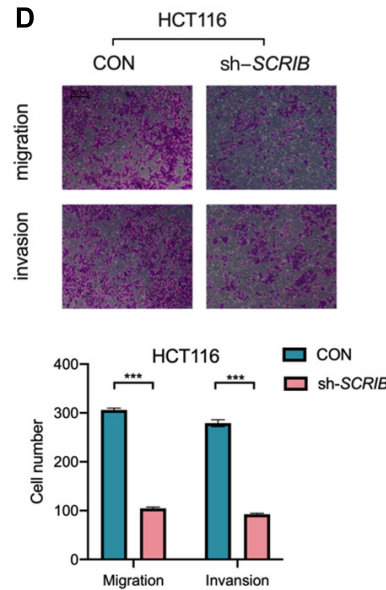

$\mathbf{F}$

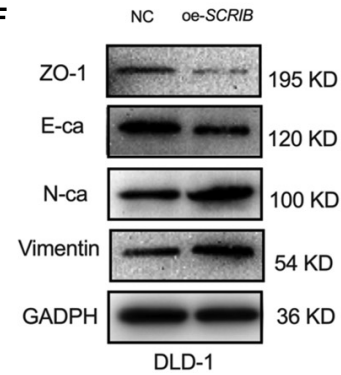

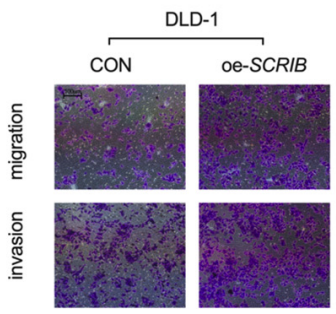

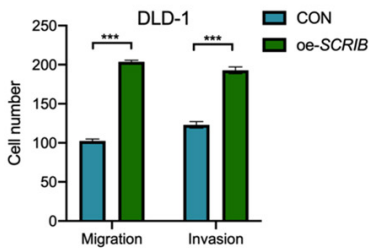

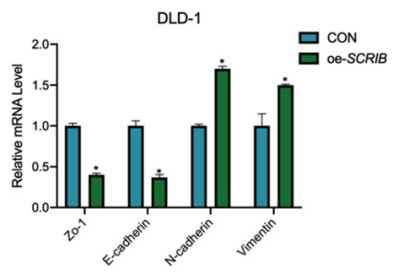

FIGURE 2 | SCRIB promotes the migration and invasion of CRC cells in vitro. SCRIB was knocked down and overexpressed in HCT116 and DLD-1 cells, respectively. The efficiency of knockdown and overexpression was verified by qRT-PCR and WB (A,B). (C) Effects of SCRIB on migration in CRC cell lines were detected by wound healing assay, scale bars: $100 \mu \mathrm{m}$. (D) Cell migration and invasion of HCT116 and DLD-1 cells as measured by Transwell assays; scale bars: $100 \mu \mathrm{m}$. (E) Western blot analysis of the tight junction protein ZO-1, epithelial marker E-cadherin, and mesenchymal markers N-cadherin and Vimentin after SCRIB knockdown in HCT116 cells. (F) The same molecules were observed in DLD-1 cells overexpressing SCRIB. The data represent the mean \pm SD from three independent experiments. Student's $t$-test was used to determine statistical significance: ${ }^{*} P<0.05,{ }^{* *} P<0.01$, and ${ }^{* \star *} P<0.001$.

(p-YAP) increased or decreased accordingly (Figure 5E). In addition, the cytoplasm/nuclear protein extraction assay showed that the nuclear accumulation of YAP in SCRIB-knockdown HCT116 cells was lower than that in control cells, while the nuclear accumulation of YAP in SCRIB-overexpressing DLD1 cells was greater than that in control cells (Figure 5F). The IF assay showed that YAP in HCT116 cells mainly accumulates in the nucleus, but the knockdown of SCRIB maintains YAP in the cytoplasm. The overexpression of SCRIB in DLD-1 cells significantly increased the level of YAP in the nucleus (Figure 5G). IHC of the CRC specimens revealed a positive correlation between SCRIB expression and YAP nuclear localization (Figure 5H).

\section{Agonists and Inhibitors of the Hippo \\ Signalling Pathway Can Restore SCRIB-Mediated CRC Cell Proliferation, Invasion and Migration}

XMU-MP-1 is a reversible and selective MST1/2 inhibitor that promotes YAP translocation to the nucleus by reducing the phosphorylation of YAP (Fan et al., 2016). Kil6425 
A

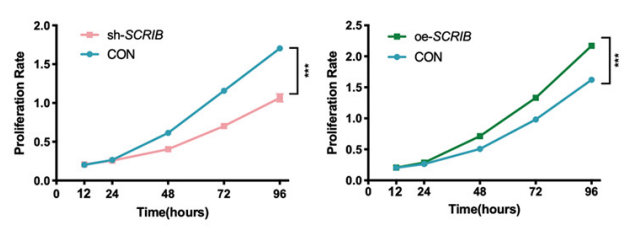

C

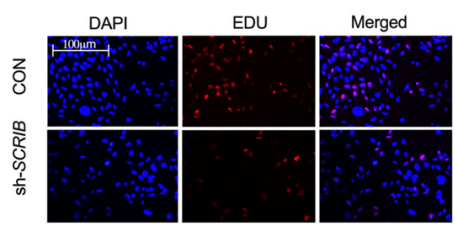

D

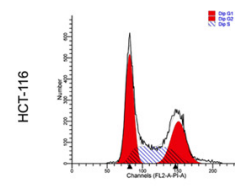

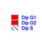

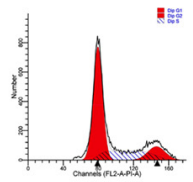

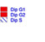

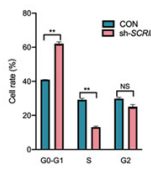

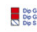
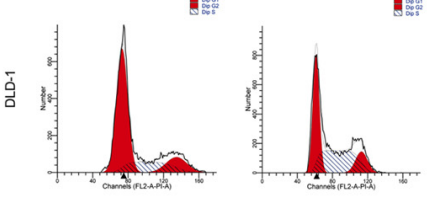

음

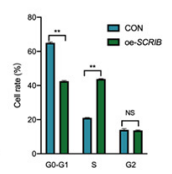

B
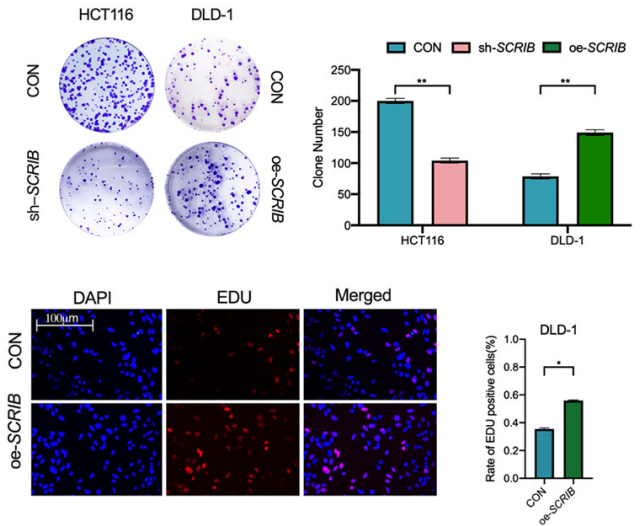

E
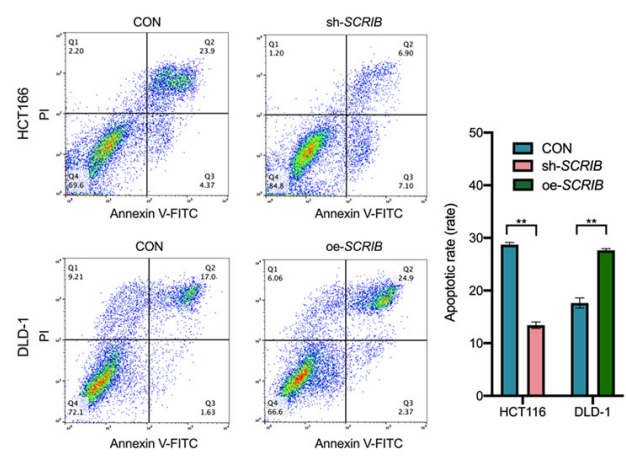

F

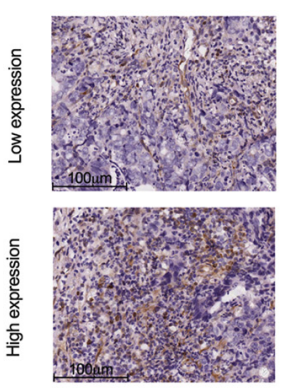

SCRIB

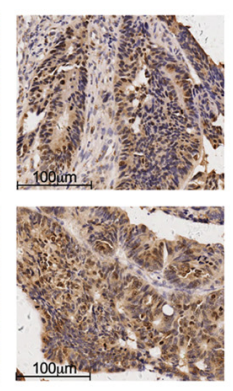

Ki-67

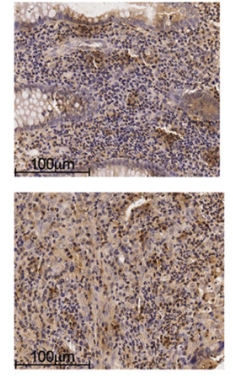

CDK4

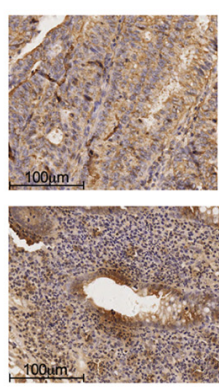

Bax

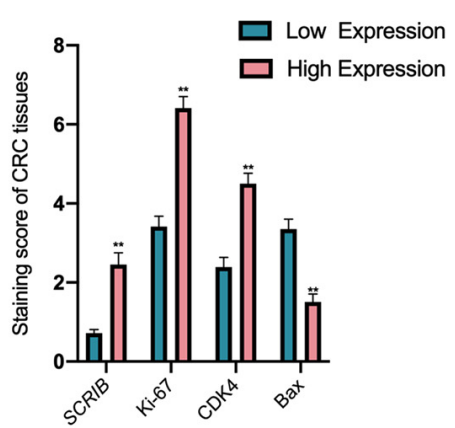

FIGURE 3 | SCRIB promotes CRC cell proliferation and inhibits apoptosis in vitro. (A) Cells were seeded in 96-well plates after transfection with oe-SCRIB and sh-SCRIB and the corresponding empty vectors, and the cell number was assessed on days 1-4 using CCK-8 assays. The knockdown of SCRIB suppressed the proliferation of HCT116 cells, whereas the overexpression of SCRIB promoted the proliferation of DLD-1 cells. (B) Effect of overexpression or knockdown of SCRIB on the colony formation ability of CRC cells; representative graphs are shown. (C) EdU immunofluorescence staining confirmed the function of SCRIB in the proliferation of CRC cells, scale bars: $100 \mu \mathrm{m}$. (D) Flow cytometry showing significant increases or decreases in the proportion of HCT116 cells in the G1 or S phase, respectively, when SCRIB was knocked down. In the SCRIB-overexpressing DLD-1 cells, the number of cells in the G1 or S phase decreased or increased, respectively. (E) The apoptotic rates (LR + UR) of transfected cells were detected by flow cytometry (Q2 + Q3). LR, early apoptotic cells; UR, terminal apoptotic cells. (F) Immunohistochemical staining of Ki-67, CDK4 and Bax was performed, and their expression was compared between SCRIB high-expressing CRC tissues and SCRIB low-expressing tissues, scale bars: $100 \mu \mathrm{m}$. The data are shown as the mean \pm SD of three independent experiments, ${ }^{\star} P<0.05$, ${ }^{\star \star} P<0.01$, and ${ }^{\star * \star} P<0.001$.

inhibits the dephosphorylation of YAP induced by serum-borne lysophosphatidic acid (LPA) and thus reduces the amount of YAP in the nucleus (Yu et al., 2012). We used these two drugs to determine that the effect of SCRIB on the malignant phenotype of CRC cells is dependent on YAP. To a certain extent, the use of these drugs reversed the phosphorylation status of YAP, which changes with the expression of SCRIB (Figure 6A). An immunofluorescence experiment also showed that the accumulation of YAP in the cytoplasm was no longer changed due to the use of pathway agonists and inhibitors (Figure 6B). In addition, drug treatment eliminated the SCRIBmediated increase in cell proliferation, invasion and migration 
A

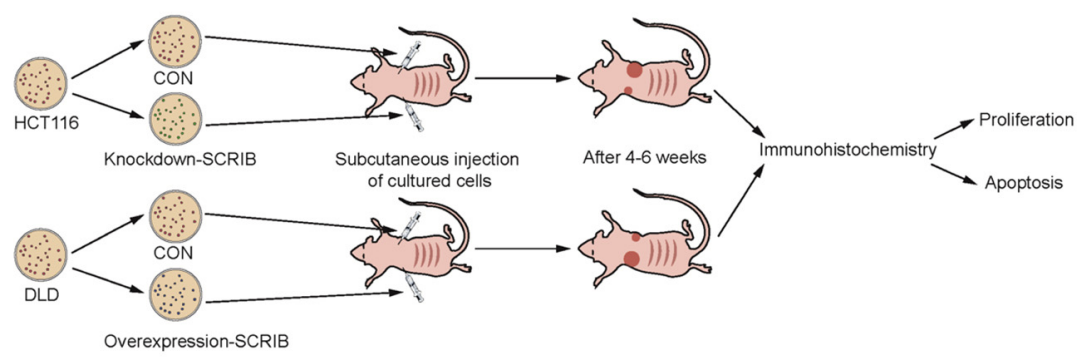

B
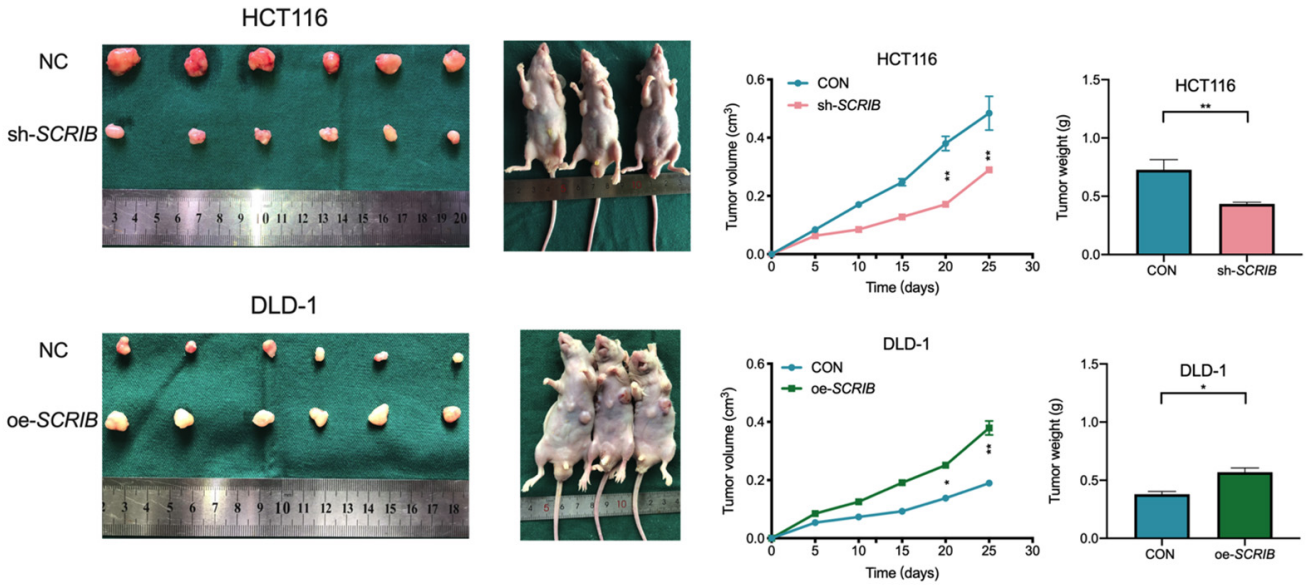

C
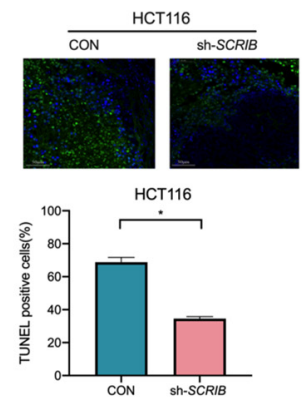
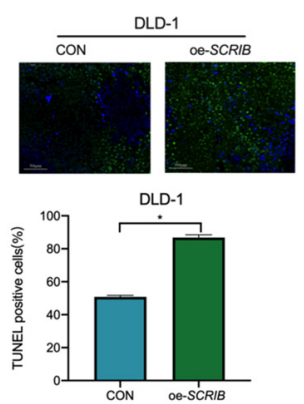

D
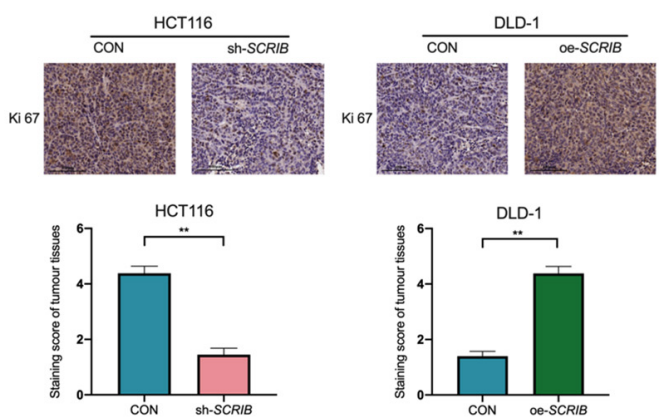

FIGURE 4 | SCRIB enhances tumour growth in vivo. (A) Nude mice were subcutaneously injected with HCT116 cells transfected with lentivirus and DLD-1 cells transfected with overexpression plasmid and their corresponding control cells. The established tumours in the right and left groins of the mice were measured every 5 days, and the mice were sacrificed 4-6 weeks after implantation. (B) Knockdown of SCRIB inhibited the tumour growth of HCT116 cells in nude mice, while overexpression of SCRIB promoted the tumour growth of DLD-1 cells in nude mice. (C) Apoptotic cells in tumour tissue were detected by TUNEL assay, and the number of TUNEL-positive cells was quantified; scale bars: $50 \mu \mathrm{m}$. (D) Protein levels of Ki67 in the tumour samples were determined by IHC, scale bars: The data are shown as the mean $\pm \mathrm{SD}$ of three independent experiments, ${ }^{*} P<0.05,{ }^{* *} P<0.01$.

(Figures 6C-G). In summary, these results suggest that YAP is essential for the $S C R I B$-mediated malignant phenotype of CRC cells.

\section{DISCUSSION}

In this study, we focused our work on the SCRIB-mediated regulation of CRC progression. Our research started by verifying the expression level of $S C R I B$ in CRC tissues, including an analysis of the available data in the TCGA database and verification with samples from our laboratory. We have discussed in our previous works that the trend of SCRIB expression in different tumours may be different, even opposite (Shen et al., 2020). Although we previously predicted that SCRIB may have a significant impact on the development of CRC, it has not been experimentally confirmed. In this study, we not only determined that SCRIB is highly expressed in CRC tissues and promotes the progression of CRC but also further studied the mechanism.

The establishment of cell polarity and intercellular adhesion lead to high-order structural organization, and the disruption or complete loss of this organization is often accompanied by neoplastic transformation (Lee and Vasioukhin, 2008). However, the regulation of cell polarity is extremely complicated. On the 
A

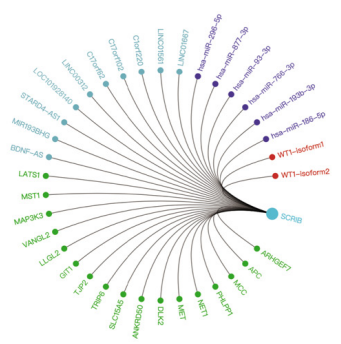

D

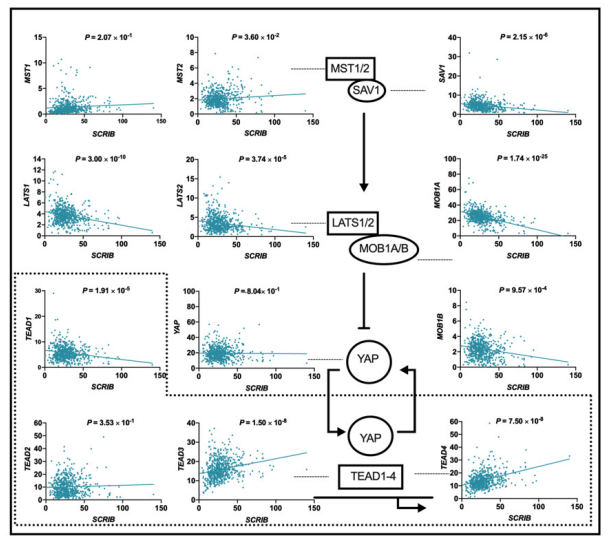

G
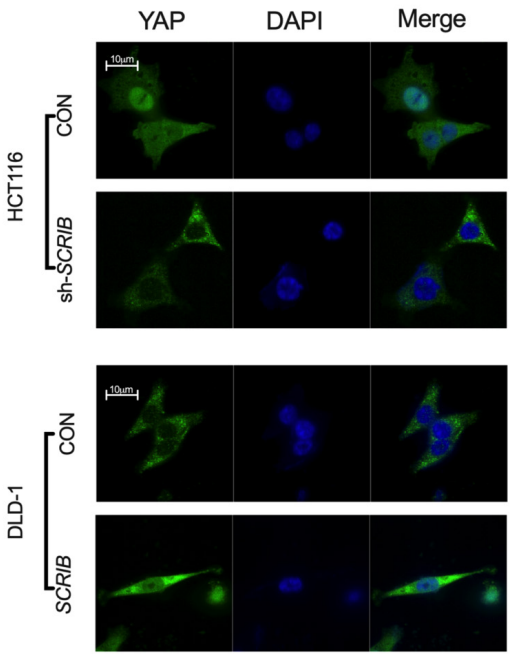

B

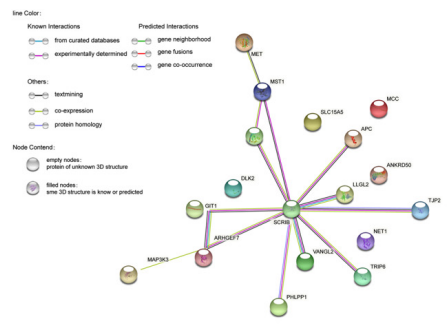

E
C

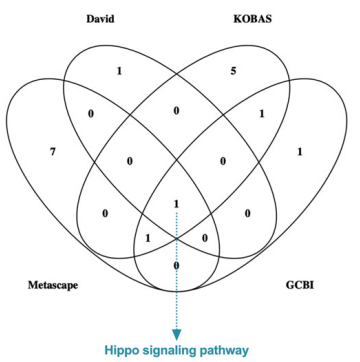

DLD-1

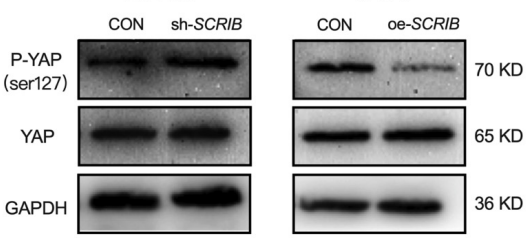

F

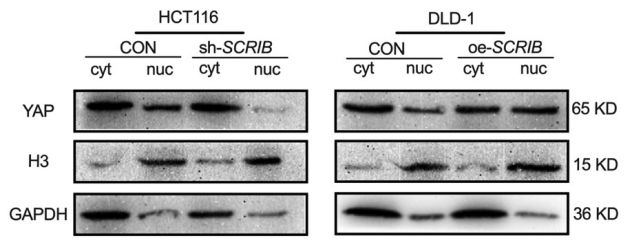

H

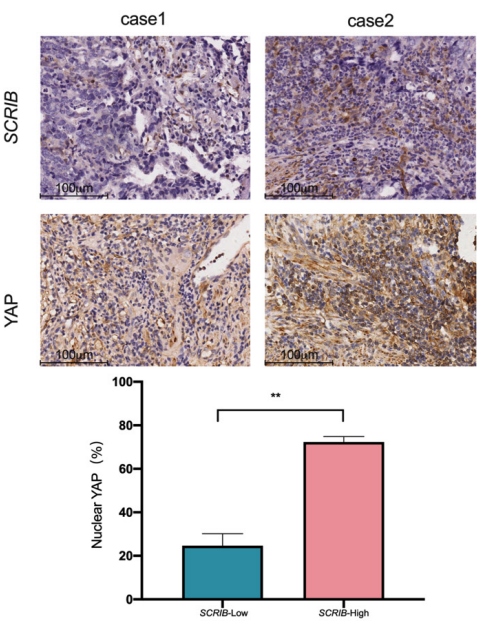

FIGURE 5 || SCRIB promotes the Hippo/YAP signalling pathway in CRC cells. (A) SCRIB-related regulatory network. Green represents interacting proteins, light blue represents related IncRNAs, purple represents targeted miRNAs, and red represents activated transcription factors. (B) Interactions between proteins predicted by the STRING database. The empty nodes represent the 3D structure position of the protein, and the filled nodes represent the known or predicted 3D structure of the protein. Proteins determined to be related to each other are connected by light blue and dark purple, and these relations have been verified by databases and experiments, respectively. Among the predicted protein interactions, dark green represents the gene neighbourhood, red represents gene fusions, and dark blue represents gene cooccurrence. The remaining lines represent weaker connections between proteins: light green represents text mining, black represents coexpression, and light purple represents protein homology. (C) David, KOBAS, Metascape and GCBI were used to predict the downstream pathways of SCRIB, and the Hippo signalling pathway was the only signalling pathway identified in all four databases. (D) The correlation between SCRIB and the core genes of the Hippo signalling pathway was analysed through the TCGA database. The dashed box indicates the nucleus. A straight arrow indicates activation, and a straight blunt arrow indicates inhibition. (E) Nuclear and cytoplasmic protein separation from the indicated CRC cells was performed to detect the expression of YAP by western blotting. Histone H3 and GAPDH were used as the loading controls for nuclear proteins and cytoplasmic proteins, respectively. (F) Cell lysates from the indicated cells were subjected to immunoblotting for phosphorylated YAP (Ser127) and total YAP. (G) Representative images showing the influence of different expression levels of SCRIB on the intracellular distribution of YAP; scale bar: $10 \mu \mathrm{m}$. (H) Representative images of immunohistochemical staining for SCRIB and YAP in sections from CRC tumours, scale bar: $100 \mu \mathrm{m} .{ }^{\star \star} P<0.01$. 
A
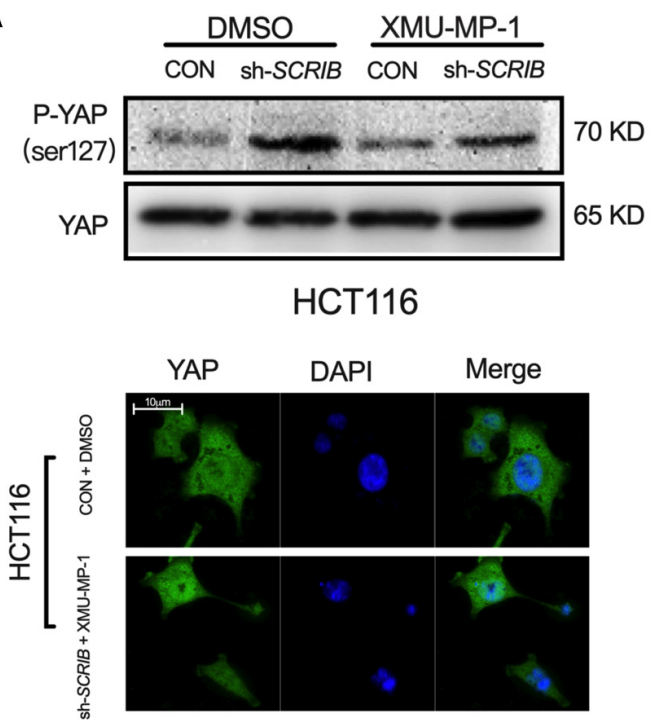

C
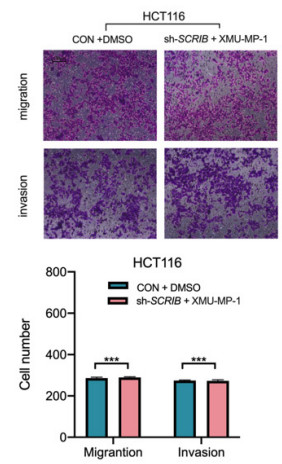

E
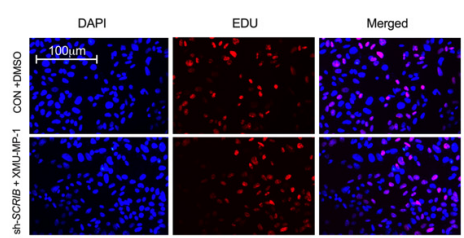

DAPI

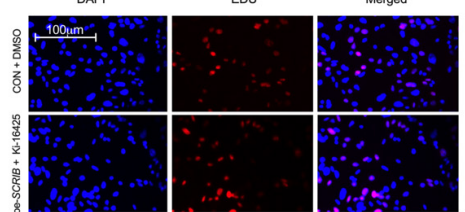

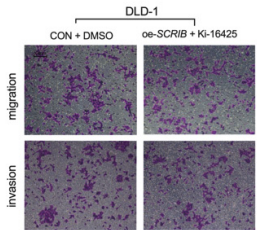

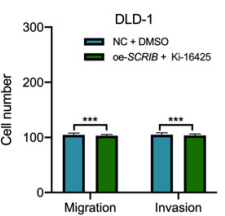

B

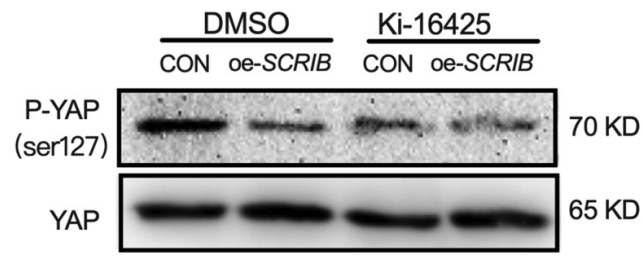

DLD-1

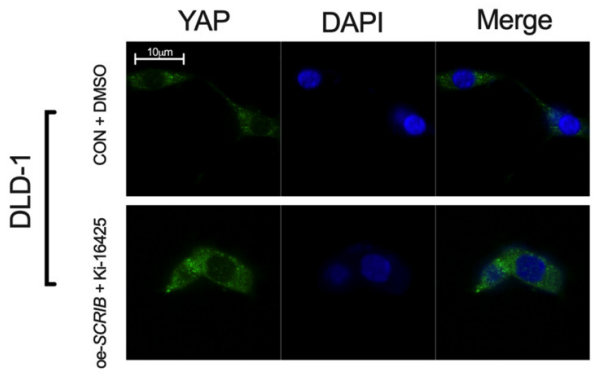

D
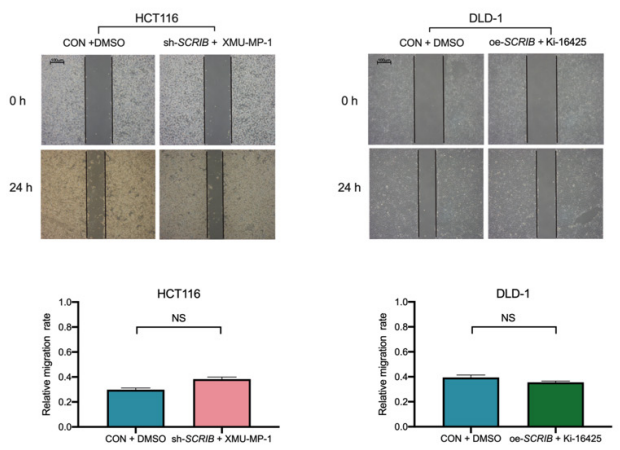

F
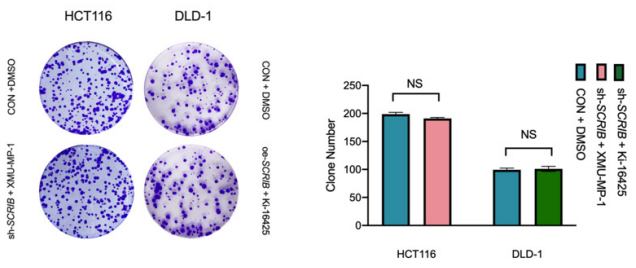

G

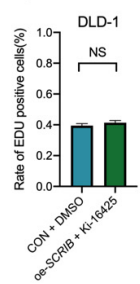

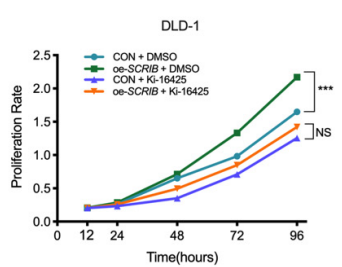

FIGURE 6 | Agonists and inhibitors of the Hippo signalling pathway can restore SCRIB-mediated CRC cell proliferation, invasion and migration. Hippo signalling pathway agonists and inhibitors were used to treat the indicated CRC cells, and the corresponding control cells were treated with DMSO to eliminate errors caused by the drugs. (A) Western blotting was used to detect the phosphorylated YAP (Ser127) and total YAP levels after the cells were treated. (B) Representative images and quantification of immunofluorescence staining for YAP distribution after cells were treated with XMU-MP-1 (1 $\mu$ M) and Ki-16425 (2 $\mu$ M) for 12 h. (C,D) Cell invasion and migration were detected by Transwell assay and scratch wound healing assay at various time points (ns: no significance). (E-G) Cell proliferation was detected by EdU, colony formation and CCK-8 assays at various time points ( ${ }^{\star \star \star} P<0.001$, ns: no significance). 


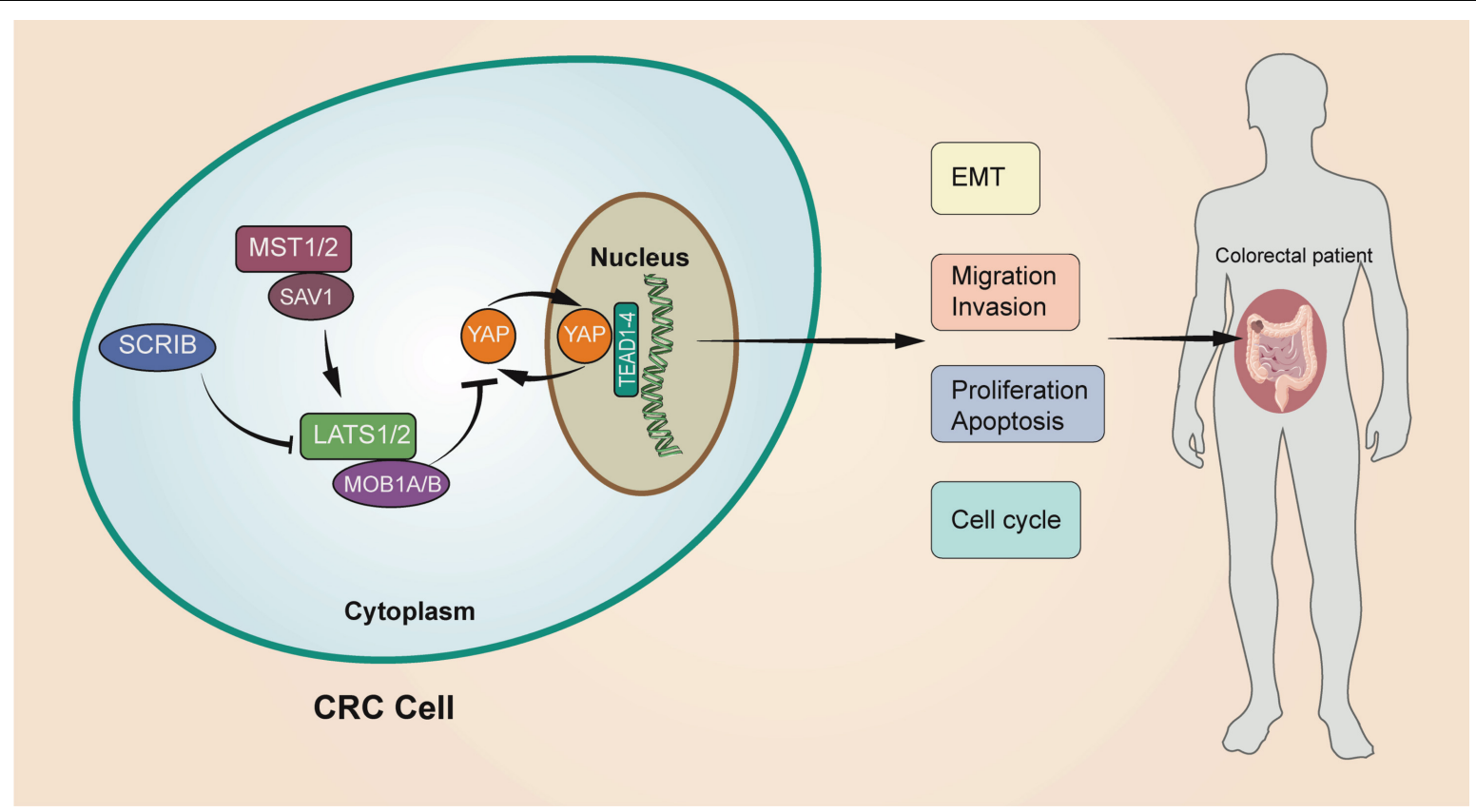

FIGURE 7 | A schematic model of the role of SCRIB in regulating CRC. The upregulation of SCRIB reduced the expression of LATS1/2 and MOB1A/B to further inhibit the phosphorylation of YAP. Unphosphorylated YAP can translocate to the nucleus to activate transcription of the target genes TEAD1-4. The SCRIB-YAP-Hippo axis ultimately affects the disease progression of CRC patients by affecting the proliferation, invasion, migration, and apoptosis of CRC cells.

apical and basal sides of cells, different polar protein complexes antagonize each other to maintain cell polarity (Bilder, 2004; Margolis and Borg, 2005; Suzuki and Ohno, 2006). A large number of studies have found that the expression of the ScribLgl-Dlg polar protein complex is reduced or completely lost in the primary tumours of human patients (Cavatorta et al., 2004; Navarro et al., 2005; Gardiol et al., 2006; Kuphal et al., 2006). However, the conclusion that the deletion of the cell polarity genes $\mathrm{dlg}$, lgl or scib can cause tumours in Drosophila is not completely applicable to mammals. Some studies have shown that the deletion of these genes in mammals may only cause asymmetric cell division and not cancer (Klezovitch et al., 2004; Mahoney et al., 2006; Cuthbert et al., 2007). One of the reasons may be that compared with Drosophila, in the mammalian system, the core cell polarity components exhibit large amounts of redundancy, so the mutation of a single gene is not enough to cause tumours. In our study, we concluded that the increased expression of SCRIB promotes the progression of CRC by affecting proliferation, invasion, metastasis, and apoptosis. It is worth noting that during our research, we found that with the increase in SCRIB, the expression of E-cadherin and ZO-1 was downregulated and the expression of vimentin and $\mathrm{N}$-cadherin, which are key epithelial markers of EMT, was upregulated. In the EMT process, epithelial cells acquire invasive capacities, mainly through the weakening of cell-cell junctions and the rearrangement of the cytoskeleton. However, before that, the first event that occurs in epithelial cells is the loss of apical-basal polarity (Kalluri and Weinberg, 2009; De Craene and Berx, 2013; Lamouille et al., 2014). The study by Jung et al. (2019) explained that apical-basal polarity can directly affect the EMT process to inhibit invasion and metastasis and even serve as a critical checkpoint. Wan et al. (2018) found that SCRIB was overexpressed in human liver cancer cells, and the dysregulation of SCRIB may play an important role in hepatocarcinogenesis and HCC cell dissemination.

An interaction between SCRIB and the Hippo signalling pathway has been reported (Enomoto and Igaki, 2011; MartinBelmonte and Perez-Moreno, 2011), and this interaction was used as the basis for listing SCRIB as a gene related to the Hippo signalling pathway in our previous studies (Shen et al., 2020). In this study, we again identified the Hippo signalling pathway as the pathway that is most closely related to SCRIB through bioinformatics analysis. Our work found that YAP, the core molecule of the Hippo signalling pathway, did not change with increasing SCRIB expression. However, the expression levels of genes targeted by YAP in the nucleus increased accordingly. Considering that YAP affects downstream target genes by entering the nucleus, we hypothesized that the increased expression of SCRIB likely promotes YAP translocation to the nucleus; we subsequently confirmed this hypothesis through experiments. We found that four genes, LATS1/2 and MOB1A/B, which are closely associated with YAP and are upstream of the Hippo signalling pathway, are negatively correlated with SCRIB in terms of expression. It is well known that the Hippo signalling pathway functions to phosphorylate YAP through a series of upstream phosphorylation cascades. This process prevents YAP from entering the nucleus and ultimately controls excessive cell growth (Zhao et al., 2007; Martin-Belmonte and Perez-Moreno, 2011). 
Combining our research results, we can conclude that the high expression of SCRIB in CRC tissues downregulates the LATS1/2 and MOB1A/B genes, reducing the phosphorylation of YAP, increasing its translocation to the nucleus, and ultimately activating nuclear oncogenes.

Our research still has some limitations. First, the number of CRC tissue samples used was not large, which makes our correlation analysis of the expression of SCRIB and the pathological characteristics of CRC patients have a certain deviation. On the other hand, due to the limitation of the experimental conditions, the specific mechanism by which SCRIB affects YAP is not detailed. How genes upstream of the Hippo signalling pathway, such as LATS1/2, participate in the process by which $S C R I B$ regulates YAP still requires further exploration.

In conclusion, we verified that SCRIB is highly expressed in $\mathrm{CRC}$ and participates in tumour progression through in vivo and in vitro experiments. In addition, we explained that the effect of SCRIB on colorectal cancer is achieved by affecting the nuclear translocation of YAP, the core molecule of the Hippo signalling pathway (Figure 7). Our work complements the study of the interaction between polar proteins and the Hippo signalling pathway and provides a possibility of identifying new therapeutic targets for CRC.

\section{DATA AVAILABILITY STATEMENT}

The datasets presented in this study can be found in online repositories. The names of the repository/repositories and accession number(s) can be found in the article/ Supplementary Material.

\section{REFERENCES}

Assémat, E., Bazellières, E., Pallesi-Pocachard, E., Le Bivic, A., and MasseyHarroche, D. (2008). Polarity complex proteins. Biochim. Biophys. Acta 1778, 614-630. doi: 10.1016/j.bbamem.2007.08.029

Beckers, R. C. J., Lambregts, D. M. J., Lahaye, M. J., Rao, S. X., Kleinen, K., Grootscholten, C., et al. (2018). Advanced imaging to predict response to chemotherapy in colorectal liver metastases-a systematic review. HPB (Oxford) 20, 120-127. doi: 10.1016/j.hpb.2017.10.013

Bilder, D. (2004). Epithelial polarity and proliferation control: links from the Drosophila neoplastic tumor suppressors. Genes Dev. 18, 1909-1925. doi: 10. 1101/gad.1211604

Cavatorta, A. L., Fumero, G., Chouhy, D., Aguirre, R., Nocito, A. L., Giri, A. A., et al. (2004). Differential expression of the human homologue of drosophila discs large oncosuppressor in histologic samples from human papillomavirusassociated lesions as a marker for progression to malignancy. Int. J. Cancer 111, 373-380. doi: 10.1002/ijc.20275

Chatterjee, S. J., and McCaffrey, L. (2014). Emerging role of cell polarity proteins in breast cancer progression and metastasis. Breast Cancer (Dove Med. Press) 6, 15-27. doi: 10.2147/bctt.S43764

Cuthbert, P. C., Stanford, L. E., Coba, M. P., Ainge, J. A., Fink, A. E., Opazo, P., et al. (2007). Synapse-associated protein 102/dlgh3 couples the NMDA receptor to specific plasticity pathways and learning strategies. J. Neurosci. 27, 2673-2682. doi: 10.1523/jneurosci.4457-06.2007

De Craene, B., and Berx, G. (2013). Regulatory networks defining EMT during cancer initiation and progression. Nat. Rev. Cancer. 13, 97-110. doi: 10.1038/ $\operatorname{nrc} 3447$

\section{ETHICS STATEMENT}

The studies involving human participants were reviewed and approved by Ethics Committee of The First Affiliated Hospital of Nanjing Medical University. The patients/participants provided their written informed consent to participate in this study. The animal study was reviewed and approved by Committee on the Ethics of Animal Experiments of Nanjing Medical University.

\section{AUTHOR CONTRIBUTIONS}

HS performed molecular biology experiment and write the main manuscript. ZF designed the whole project and supervised all experiments. $\mathrm{CH}, \mathrm{JW}$, and $\mathrm{JL}$ conducted all experiments and analysed the data. TH, ZW, HZ, and YS provided support with experimental and clinical techniques. All authors read and approved the final manuscript.

\section{FUNDING}

This work was supported by the National Natural Science Foundation of China (81470881 to ZF) and Jiangsu Commission of Health (LGY2017031 and BRA2015473 to ZF).

\section{SUPPLEMENTARY MATERIAL}

The Supplementary Material for this article can be found online at: https://www.frontiersin.org/articles/10.3389/fcell.2021. 656359/full\#supplementary-material

Enomoto, M., and Igaki, T. (2011). Deciphering tumor-suppressor signaling in flies: genetic link between Scribble/Dlg/Lgl and the Hippo pathways. J. Genet. Genomics 38, 461-470. doi: 10.1016/j.jgg.2011.09.005

Fan, F., He, Z., Kong, L. L., Chen, Q., Yuan, Q., Zhang, S., et al. (2016). Pharmacological targeting of kinases MST1 and MST2 augments tissue repair and regeneration. Sci. Transl. Med. 8:352ra108. doi: 10.1126/scitranslmed. aaf 2304

Feigin, M. E., Akshinthala, S. D., Araki, K., Rosenberg, A. Z., Muthuswamy, L. B., Martin, B., et al. (2014). Mislocalization of the cell polarity protein scribble promotes mammary tumorigenesis and is associated with basal breast cancer. Cancer Res. 74, 3180-3194. doi: 10.1158/0008-5472.Can-13-3415

Gardiol, D., Zacchi, A., Petrera, F., Stanta, G., and Banks, L. (2006). Human discs large and scrib are localized at the same regions in colon mucosa and changes in their expression patterns are correlated with loss of tissue architecture during malignant progression. Int. J. Cancer 119, 1285-1290. doi: 10.1002/ijc. 21982

Hanahan, D., and Weinberg, R. A. (2000). The hallmarks of cancer. Cell 100, 57-70. doi: 10.1016/s0092-8674(00)81683-9

Hanahan, D., and Weinberg, R. A. (2011). Hallmarks of cancer: the next generation. Cell 144, 646-674. doi: 10.1016/j.cell.2011.02.013

Hu, T., Shen, H., Li, J., Yang, P., Gu, Q., and Fu, Z. (2020). RFC2, a direct target of miR-744, modulates the cell cycle and promotes the proliferation of CRC cells. J. Cell. Physiol. 235, 8319-8333. doi: 10.1002/jcp.29676

Jung, H. Y., Fattet, L., Tsai, J. H., Kajimoto, T., Chang, Q., Newton, A. C., et al. (2019). Apical-basal polarity inhibits epithelial-mesenchymal transition and tumour metastasis by PAR-complex-mediated SNAI1 degradation. Nat. Cell Biol. 21, 359-371. doi: 10.1038/s41556-019-0291-8 
Kalluri, R., and Weinberg, R. A. (2009). The basics of epithelial-mesenchymal transition. J. Clin. Invest. 119, 1420-1428. doi: 10.1172/jci39104

Klezovitch, O., Fernandez, T. E., Tapscott, S. J., and Vasioukhin, V. (2004). Loss of cell polarity causes severe brain dysplasia in Lgl1 knockout mice. Genes Dev. 18, 559-571. doi: 10.1101/gad.1178004

Kuphal, S., Wallner, S., Schimanski, C. C., Bataille, F., Hofer, P., Strand, S., et al. (2006). Expression of Hugl-1 is strongly reduced in malignant melanoma. Oncogene 25, 103-110. doi: 10.1038/sj.onc. 1209008

Lamouille, S., Xu, J., and Derynck, R. (2014). Molecular mechanisms of epithelialmesenchymal transition. Nat. Rev. Mol. Cell Biol. 15, 178-196. doi: 10.1038/ nrm3758

Lee, M., and Vasioukhin, V. (2008). Cell polarity and cancer-cell and tissue polarity as a non-canonical tumor suppressor. J. Cell Sci. 121(Pt 8), 1141-1150. doi: $10.1242 /$ jcs. 016634

Mahoney, Z. X., Sammut, B., Xavier, R. J., Cunningham, J., Go, G., Brim, K. L., et al. (2006). Discs-large homolog 1 regulates smooth muscle orientation in the mouse ureter. Proc. Natl. Acad. Sci. U.S.A. 103, 19872-19877. doi: 10.1073/pnas. 0609326103

Margolis, B., and Borg, J. P. (2005). Apicobasal polarity complexes. J. Cell Sci. 118(Pt 22), 5157-5159. doi: 10.1242/jcs.02597

Martin-Belmonte, F., and Perez-Moreno, M. (2011). Epithelial cell polarity, stem cells and cancer. Nat. Rev. Cancer 12, 23-38. doi: 10.1038/nrc3169

Muthuswamy, S. K., and Xue, B. (2012). Cell polarity as a regulator of cancer cell behavior plasticity. Annu. Rev. Cell Dev. Biol. 28, 599-625. doi: 10.1146/ annurev-cellbio-092910-154244

Navarro, C., Nola, S., Audebert, S., Santoni, M. J., Arsanto, J. P., Ginestier, C., et al. (2005). Junctional recruitment of mammalian Scribble relies on E-cadherin engagement. Oncogene 24, 4330-4339. doi: 10.1038/sj.onc. 1208632

Rothenberg, S. M., Mohapatra, G., Rivera, M. N., Winokur, D., Greninger, P., Nitta, M., et al. (2010). A genome-wide screen for microdeletions reveals disruption of polarity complex genes in diverse human cancers. Cancer Res. 70, 2158-2164. doi: 10.1158/0008-5472.Can-09-3458

Royer, C., and Lu, X. (2011). Epithelial cell polarity: a major gatekeeper against cancer? Cell Death Differ. 18, 1470-1477. doi: 10.1038/cdd.2011.60

Shen, H., Meng, Y., Hu, T., Li, S., Du, M., Xin, J., et al. (2020). Genetic variants in Hippo signalling pathway-related genes affect the risk of colorectal cancer. Arch. Toxicol. 95, 271-281. doi: 10.1007/s00204-020-02910-3

Siegel, R. L., Miller, K. D., Goding Sauer, A., Fedewa, S. A., Butterly, L. F., Anderson, J. C., et al. (2020a). Colorectal cancer statistics, 2020. CA Cancer J. Clin. 70, 145-164. doi: 10.3322/caac. 21601
Siegel, R. L., Miller, K. D., and Jemal, A. (2020b). Cancer statistics, 2020. CA Cancer J. Clin. 70, 7-30. doi: 10.3322/caac. 21590

St Johnston, D., and Ahringer, J. (2010). Cell polarity in eggs and epithelia: parallels and diversity. Cell 141, 757-774. doi: 10.1016/j.cell.2010.05.011

Suzuki, A., and Ohno, S. (2006). The PAR-aPKC system: lessons in polarity. J. Cell Sci. 119(Pt 6), 979-987. doi: $10.1242 /$ jcs.02898

Wan, S., Meyer, A. S., Weiler, S. M. E., Rupp, C., Tóth, M., Sticht, C., et al. (2018). Cytoplasmic localization of the cell polarity factor scribble supports liver tumor formation and tumor cell invasiveness. Hepatology 67, 1842-1856. doi: 10.1002/hep.29669

Yu, F. X., Zhao, B., Panupinthu, N., Jewell, J. L., Lian, I., Wang, L. H., et al. (2012). Regulation of the Hippo-YAP pathway by G-protein-coupled receptor signaling. Cell 150, 780-791. doi: 10.1016/j.cell.2012.06.037

Zen, K., Yasui, K., Gen, Y., Dohi, O., Wakabayashi, N., Mitsufuji, S., et al. (2009). Defective expression of polarity protein PAR-3 gene (PARD3) in esophageal squamous cell carcinoma. Oncogene 28, 2910-2918. doi: 10.1038/onc.20 09.148

Zhan, L., Rosenberg, A., Bergami, K. C., Yu, M., Xuan, Z., Jaffe, A. B., et al. (2008). Deregulation of scribble promotes mammary tumorigenesis and reveals a role for cell polarity in carcinoma. Cell 135, 865-878. doi: 10.1016/j.cell.2008. 09.045

Zhao, B., Wei, X., Li, W., Udan, R. S., Yang, Q., Kim, J., et al. (2007). Inactivation of YAP oncoprotein by the Hippo pathway is involved in cell contact inhibition and tissue growth control. Genes Dev. 21, 2747-2761. doi: 10.1101/gad.16 02907

Zhu, J., Tan, Z., Hollis-Hansen, K., Zhang, Y., Yu, C., and Li, Y. (2017). Epidemiological trends in colorectal cancer in China: an ecological study. Dig. Dis. Sci. 62, 235-243. doi: 10.1007/s10620-016-4362-4

Conflict of Interest: The authors declare that the research was conducted in the absence of any commercial or financial relationships that could be construed as a potential conflict of interest.

Copyright $(2021$ Shen, Huang, $W u, L i, H u$, Wang, Zhang, Shao and Fu. This is an open-access article distributed under the terms of the Creative Commons Attribution License (CC BY). The use, distribution or reproduction in other forums is permitted, provided the original author(s) and the copyright owner(s) are credited and that the original publication in this journal is cited, in accordance with accepted academic practice. No use, distribution or reproduction is permitted which does not comply with these terms. 